\title{
Chaos in magnetospheric radio emissions
}

\author{
A. C.-L. Chian ${ }^{1,2}$, E. L. Rempel ${ }^{1,2}$, and F. A. Borotto ${ }^{1,2,3}$ \\ ${ }^{1}$ World Institute for Space Environment Research - WISER, NITP/WISER, Adelaide University, Adelaide, SA 5005, \\ Australia \\ ${ }^{2}$ National Institute for Space Research - INPE, P. O. Box 515, São José dos Campos - SP, CEP 12227-010, Brazil \\ ${ }^{3}$ Universidad de Concepción, Departamento de Física, Concepción, Chile
}

Received: 15 November 2001 - Revised: 22 February 2002 - Accepted: 10 April 2002

\begin{abstract}
A three-wave model of auroral radio emissions near the electron plasma frequency was proposed by Chian et al. (1994) involving resonant interactions of Langmuir, whistler and Alfvén waves. Chaos can occur in the nonlinear evolution of this three-wave process in the magnetosphere. In particular, two types of intermittency, due to either local or global bifurcations, can be observed. We analyze the typeI Pomeau-Manneville intermittency, arising from a saddlenode bifurcation, and the crisis-induced intermittency, arising from an interior crisis associated with a global bifurcation. Examples of time series, power spectrum, phasespace trajectory for both types of intermittency are presented through computer simulations. The degree of chaoticity of this three-wave process is characterized by calculating the maximum Lyapunov exponent. We suggest that the intermittent phenomena discussed in this paper may be observed in the temporal signal of magnetospheric radio emissions.
\end{abstract}

\section{Introduction}

Nonthermal radio waves have been observed in a number of planetary magnetospheres (Chian et al., 1993). Traditionally, it is believed that the nonlinear interaction of high-frequency electron plasma waves with low-frequency density fluctuations, such as ion-acoustic waves, is the main mechanism for beam-driven turbulence, to produce nonthermal plasma emissions at the fundamental plasma frequency. Recently, we have shown that the nonlinear coupling of Langmuir waves with low-frequency magnetic field fluctuations, such as Alfvén waves, may also provide an efficient mechanism for generating magnetospheric radio waves (Chian et al., 1994; Lopes and Chian, 1996a; Chian et al., 2000). Evidence of this emission mechanism has been obtained in rocket experiments in the Earth's auroral plasmas (Boehm et al., 1990; Chian et al., 1994). In this paper, we present a nonlinear

Correspondence to: A. C.-L. Chian

(achian@physics.adelaide.edu.au) dynamical theory of three-wave interactions involving Langmuir, Alfvén and whistler waves in the planetary magnetospheres. By assuming linear growth for the Langmuir wave and linear damping for both the Alfvén and whistler waves, this wave triplet is shown to evolve from order to chaos via a number of different routes. We identify two types of intermittency in a given periodic window of the bifurcation diagram: type-I Pomeau-Manneville intermittency and crisis-induced intermittency. Numerical solutions of these nonlinear dynamical phenomena are presented, showing the time series of the wave amplitude and the corresponding power spectrum and trajectory in the phase space. The characterization of ordered and chaotic states is performed by calculating the largest Lyapunov exponent. The relevance of this theory for detecting chaos in the time series of magnetospheric radio waves is discussed.

\section{Nonlinear coupled wave equations}

Consider the nonlinear parametric interaction of Langmuir (L), whistler (W) and Alfvén (A) waves, all propagating along the ambient magnetic field $\mathbf{B}=B_{0} \hat{\mathbf{z}}$. The three-wave resonant interaction occurs if the phase-matching condition and wave helicity conservation condition are satisfied. We assume the following phase-matching condition

$\omega_{L} \approx \omega_{W}+\omega_{A}$,

$\mathbf{k}_{L}=\mathbf{k}_{W}+\mathbf{k}_{A}$,

where we introduce a finite frequency mismatch but assume perfect wave vector match. Since the electromagnetic whistler wave has a right-hand circular polarization, the wave helicity conservation implies that the Alfvén wave must be a shear Alfvén mode which has a left-hand circular polarization.

The set of coupled wave equations governing the nonlinear interaction $L \rightleftharpoons W+A$, derived from a two-fluid theory, is given by Chian et al. (2000) as

$$
\dot{A}_{L}=v_{L} A_{L}+A_{W} A_{A} \text {, }
$$


$\dot{A}_{W}=v_{W} A_{W}-A_{L} A_{A}^{*}$,

$\dot{A}_{A}=i \delta A_{A}+v_{A} A_{A}-A_{L} A_{W}^{*}$,

with

$A_{L}=$

$\left[\frac{c_{L W} c_{L A}}{4 k^{2}\left(v_{g A}-v\right)\left(v_{g W}-v\right)\left(\partial D_{A} / \partial \omega_{A}\right)\left(\partial D_{W} / \partial \omega_{W}\right)}\right]^{1 / 2}$

$\mathcal{E}_{L}$,

$A_{W}=$

$\left[\frac{c_{W A} c_{L W}}{4 k^{2}\left(v_{g L}-v\right)\left(v_{g A}-v\right)\left(\partial D_{L} / \partial \omega_{L}\right)\left(\partial D_{A} / \partial \omega_{A}\right)}\right]^{1 / 2}$

$\mathcal{E}_{W}$,

$A_{A}=$

$\left[\frac{c_{W A} c_{L A}}{4 k^{2}\left(v_{g L}-v\right)\left(v_{g W}-v\right)\left(\partial D_{L} / \partial \omega_{L}\right)\left(\partial D_{W} / \partial \omega_{W}\right)}\right]^{1 / 2}$

$\times \mathcal{E}_{A} \exp i \Delta t$

where $\mathcal{E}(z, t)$ is a slowly varying complex envelope of the wave electric fields

$\mathbf{E}_{\alpha}(z, t)=\frac{1}{2} \mathcal{E}_{\alpha}(z, t) \exp i \theta_{\alpha}+$ c.c.,

such that $\left|\partial_{t}^{2} \mathcal{E}_{\alpha}\right| \ll\left|\omega_{\alpha} \partial_{t} \mathcal{E}_{\alpha}\right|$ and $\left|\partial_{z}^{2} \mathcal{E}_{\alpha}\right| \ll\left|k_{\alpha} \partial_{z} \mathcal{E}_{\alpha}\right|, \theta_{\alpha}=$ $k_{\alpha} z-\omega_{\alpha} t$ is the fast-varying phase, and $\alpha=(L, W, A)$. The dispersion operators are given by

$D_{L}=-\omega_{L}^{2}+\omega_{p e}^{2}+\gamma v_{t h}^{2} k_{L}^{2}-i v_{L} \omega_{L}$,

$D_{W}=-\omega_{W}^{2}+c^{2} k_{W}^{2}+\frac{\omega_{p e}^{2} \omega_{W}}{\omega_{W}-\omega_{c e}}-i \nu_{W} \omega_{W}$,

$D_{A}=-\omega_{A}^{2}+c_{A}^{2} k_{A}^{2}-i v_{A} \omega_{A}$,

and the coupling coefficients are given by

$c_{W A}=\frac{-e \omega_{p e}^{2}}{2 m_{e}\left(\omega_{W}-\omega_{c e}\right)}\left[\frac{k_{A}}{\omega_{A}}+\frac{k_{W}\left(\omega_{W}-\omega_{c e}\right)}{\omega_{W}\left(\omega_{A}+\omega_{c e}\right)}\right]$

$c_{L A}=\left(\frac{\omega_{W}^{2}}{\omega_{L}^{2}}\right) c_{W A}$,

$c_{L W}=\left(\frac{\omega_{A}^{2} c_{A}^{2}}{\omega_{L}^{2} c^{2}}\right) c_{W A}$,

where $\omega_{p e}^{2}=\left(n_{0} e^{2} / m_{e} \varepsilon_{0}\right)^{1 / 2}$ is the electron plasma frequency, $\omega_{c e}=e B_{0} / m_{e}$ is the electron cyclotron frequency, $v_{t h}=\left(K T_{e} / m_{e}\right)^{1 / 2}$ is the electron thermal velocity, $c_{A}=$ $B_{0} /\left(\mu_{0} \rho_{0}\right)^{1 / 2}$ is the Alfvén velocity, $\gamma$ is the ratio of specific heats; the dot denotes the derivative with respect to $\tau=k(z-v t), v$ and $k$ are the arbitrary wave velocity and wave vector, respectively; the frequency mismatch parameter $\delta=\Delta /\left[k\left(v_{g A}-v\right)\right], \Delta=\omega_{L}-\omega_{W}-\omega_{A}$, where $v_{g \alpha}$ represent the group velocities; $v_{\alpha}$ represent the normalized growth/damping parameters (Chian et al., 2000). We assume the Langmuir wave is linearly unstable $\left(v_{L}>0\right)$ and set $v_{L} \equiv 1$, whereas the whistler wave and the shear Alfvén wave are linearly damped and set $v_{W}=v_{A} \equiv-v<0$.

\section{Bifurcation diagram}

The set of coupled wave Eqs. (3)-(5) exhibits rich dynamical behaviors, such as fixed point, divergence, limit cycle and strange attractor (Wersinger et al., 1980; Meunier et al., 1982; Lopes and Chian, 1996b; Lopez et al., 1998; Chian et al., 2000). The system dynamics can be studied by constructing a bifurcation diagram which shows the birth, evolution, and death of attracting sets (Alligood et al., 1996). The bifurcation diagram is determined by varying only one control parameter and keeping all other control parameters fixed. A phase diagram in terms of two control parameters, $v$ and $\delta$, depicting the overall nonlinear dynamics of threewave interactions, has been systematically obtained by Meunier et al. (1982). Based on the $(\nu, \delta)$ phase diagram of Meunier et al. (1982), we fix $\delta=2$ arbitrarily and vary the parameter $v$ in order to see how the system dynamics change as the growth/damping parameter varies. Other values of $\delta$ can be chosen, which will yield different bifurcation diagrams. For each value of the control parameter $v$, we numerically integrate Eqs. (3)- (5) by dropping the initial transient. The Poincaré points are the projections of the 3-dimensional trajectory onto the 2-dimensional Poincaré plane $\left(\left(\left|A_{L}\right|,\left|A_{W}\right|\right)\right)$. A period-2 window of the numerically computed bifurcation diagram is shown in Fig. 1a, where the Poincare points refer to the maxima of $A_{W}$. The corresponding behavior of the maximum Lyapunov exponent $\lambda_{\text {max }}$ is plotted in Fig. 1b. Chaos (aperiodic solutions) occurs when $\lambda_{\max }>0$, and order (periodic solutions) occurs when $\lambda_{\max }<0$. This periodic window indicates that a saddlenode bifurcation $(\mathrm{SN})$ takes place near $v=29.56$, where a pair of period-2 stable and unstable periodic orbits is created. The stable periodic orbit undergoes a cascade of perioddoubling bifurcations, leading to chaos. At a critical parameter, $v=v_{I C}=33.23$, the chaotic attractor collides with a period-2 unstable periodic orbit evolving from the saddlenode bifurcation, leading to an interior crisis (IC) due to a global bifurcation. Figure $1 \mathrm{~b}$ shows that to the right of the saddle-node bifurcation point $\nu_{S N}, \lambda_{\max }$ is negative (order); whereas to the left of $v_{S N}, \lambda_{\max }$ is positive (chaos). In addition, we can see from Fig. $1 \mathrm{~b}$ that in the vicinity of $v_{I C}$, the values of $\lambda_{\max }$ increase abruptly, indicating a sudden increase in the system chaoticity.

\section{Pomeau-Manneville intermittency}

In the type-I Pomeau-Manneville intermittency, the coalescence of a pair of stable and unstable limit cycles gives rise to a chaotic orbit as the control parameter $v$ reaches a critical value $v_{S N}$, where a (local) saddle-node bifurcation occurs (Manneville and Pomeau, 1979; Lopes and Chian, 1996b; Chian et al., 2000). In Fig. 2, we plot a return map for $v=29.56$, which shows that in the vicinity of a saddle-node bifurcation, the curve is nearly tangent to the bisectrix. For $v$ just slightly greater than 29.56, namely at $v=v_{S N}$, the curve is exactly tangent to the bisectrix, which leads to a saddle- 

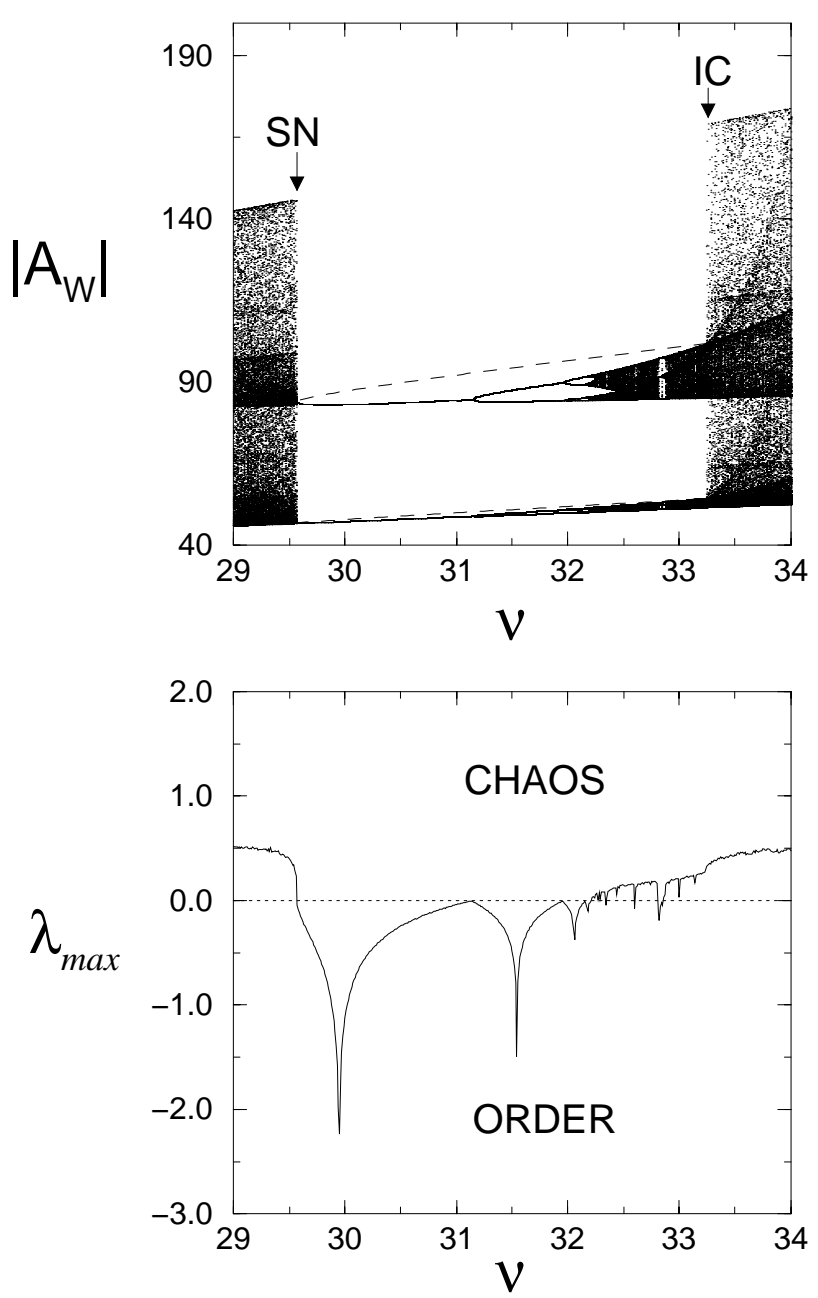

Fig. 1. (a) Bifurcation diagram for $\left|A_{W}\right|$ as a function of $v$ for a period-2 window. (b) Maximum Lyapunov exponent $\lambda_{\max }$ as a function of $v$. The dashed lines in Fig. 1a denote the period-2 unstable periodic orbit; SN denotes saddle-node bifurcation; IC denotes interior crisis.

node bifurcation due to the coalescence of a pair of unstable and stable periodic orbits of period-2 (see Fig. 11 in Chian et al., 2000). In Fig. 2, each arrow indicates an approximate site of the tangency (i.e. saddle-node bifurcation). Moreover, the occurrence of a saddle-node bifurcation at 29.56 $<v_{S N}<29.57$ can be seen by comparing Figs. 3a with $3 b$, 4a with Figs. 4b, and Figs. 4d with 4e.

In the Pomeau-Manneville intermittency of type-I, the laminar phases of nearly periodic oscillations are suddenly interrupted by chaotic bursts. This intermittency occurs in the transition region of the saddle-node bifurcation when $v<v_{S N}$. An example of time series for this intermittency is given in Fig. 3. Figure 3a shows a periodic solution with period-2 when $v>v_{S N}$; Fig. $3 \mathrm{~b}$ shows an intermittent solution just to the left of $v_{S N}$; and Fig. $3 \mathrm{c}$ shows a stronger chaotic solution further away from $v_{S N}$.

The power spectra and phase-space trajectories for a given time interval corresponding to Fig. 3 are displayed in Fig. 4.

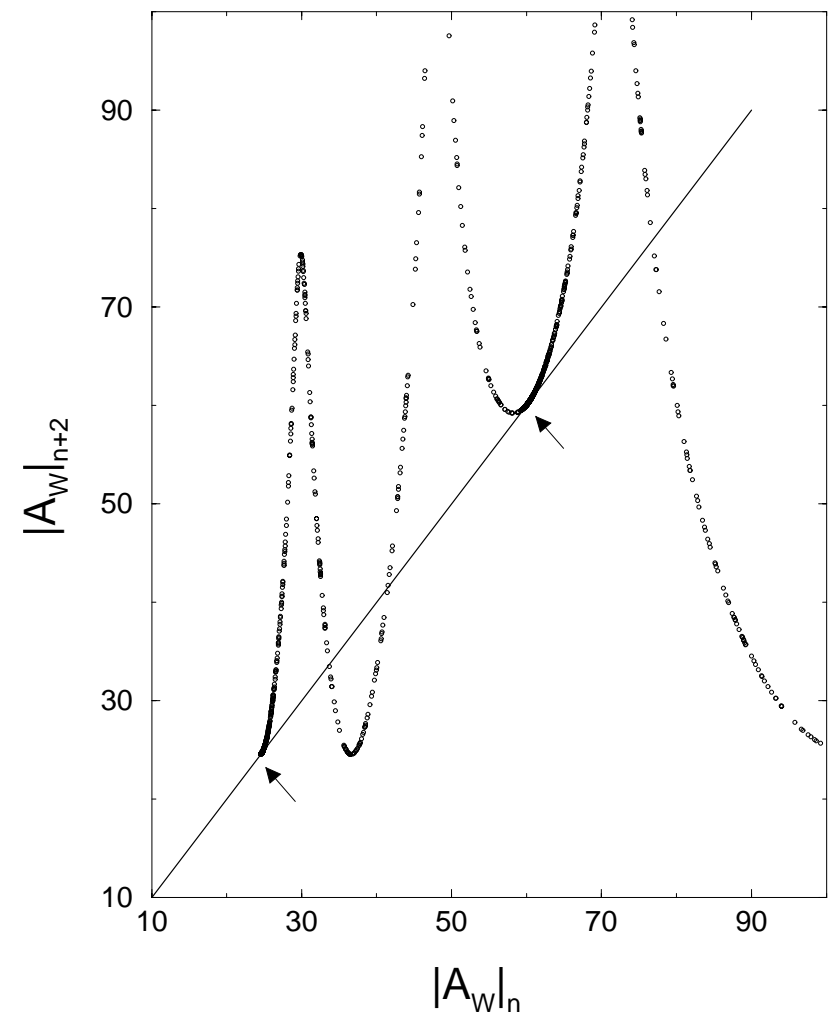

Fig. 2. Return map for the saddle-node bifurcation in the vicinity of $v=29.56$.

Figures $4 \mathrm{a}-\mathrm{c}$ illustrate the power spectra in the log-linear scale. In Fig. 4a, the power spectrum of the periodic solution at $v=29.57$ consists of discrete peaks. The corresponding period-2 attractor (limit cycle) is shown in Fig. 4d. At $v=29.56$, the power spectrum (Fig. 4b) becomes broad band and continuous, indicating the onset of chaos. Note, however, that the peaks of Fig. $4 \mathrm{a}$ are still recognizable in Fig. 4b. Figure 4e shows that, just to the left of $v_{S N}$, the orbit spends a long time close to the periodic attractor that exists when $v>v_{S N}$. At $v=29.55$, the discrete peaks of the power spectrum (Fig. 4c) decrease considerably, whereas the flucutations of the power spectrum at frequencies around the peaks increase significantly; the trajectory now spends much less time in the vicinity of the former period- 2 attractor, as seen in Fig. 4f. The changes noted in Figs. 4a-c imply that as the system evolves from order to chaos, energy is transferred from the discrete peaks to other frequencies.

The maximum Lyapunov exponents for the time series of Fig. 3 are shown in Fig. 5. For $v=29.57>v_{S N}$ the maximum Lyapunov exponent is negative, characterizing periodic behavior. The increasing chaoticity of trajectories shown in Figs. $4 \mathrm{e}$ and $4 \mathrm{f}$ is revealed by a rise in the maximum Lyapunov exponent as $v$ decreases from $v=29.56$ to $v=29.55$. 

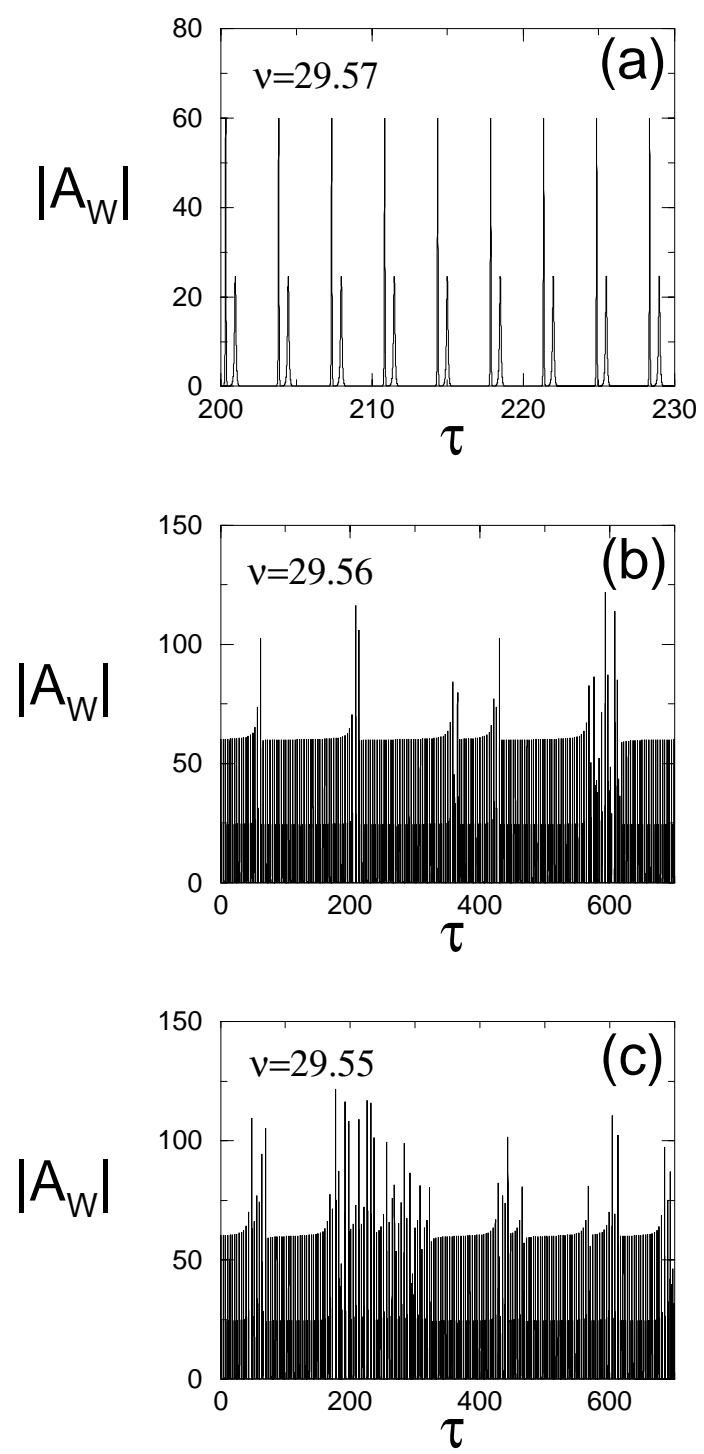

Fig. 3. Time series of $\left|A_{W}\right|$ as a function of $\tau$ for the type-I PomeauManneville intermittency route to chaos for (a) $v=29.57$, (b) $v=$ 29.56 and (c) $v=29.55$.

\section{Crisis-induced intermittency}

The crisis-induced intermittency is characterized by a time series containing "laminar" phases of weak chaotic fluctuations which are randomly interrupted by strong chaotic bursts (Grebogi et al., 1983; Chian et al., 1998). This intermittency is due to a type of global bifurcation known as interior crisis, which occurs when a weak chaotic attractor collides with an unstable periodic orbit, leading to an abrupt expansion of the size of the attractor to form a strong chaotic attractor. A Poincare plot of the chaotic attractor before, at and after the crisis point $v_{I C}$ (indicated in Fig. 1a) is given in Fig. 6, where the two crosses represent the saddle points of the period-2 unstable periodic orbit responsible for the crisis. For this figure we chose the Poincaré plane defined by $\left|A_{W}\right|=1$ in order to simplify the numerical procedure for finding the
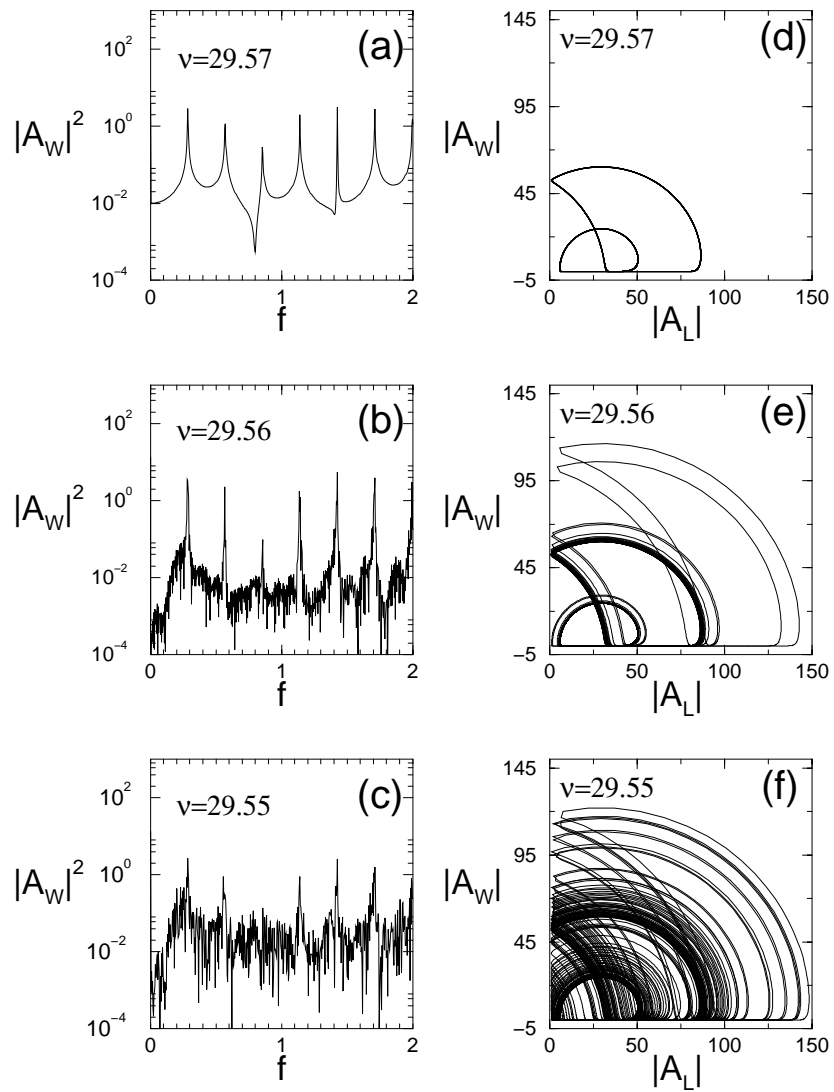

Fig. 4. Power spectra for (a) $v=29.57$, (b) $v=29.56$ and (c) $v=29.55$; with the corresponding trajectories in the phase space $\left(\left|A_{L}\right|,\left|A_{W}\right|\right)$ in (d) to (f).

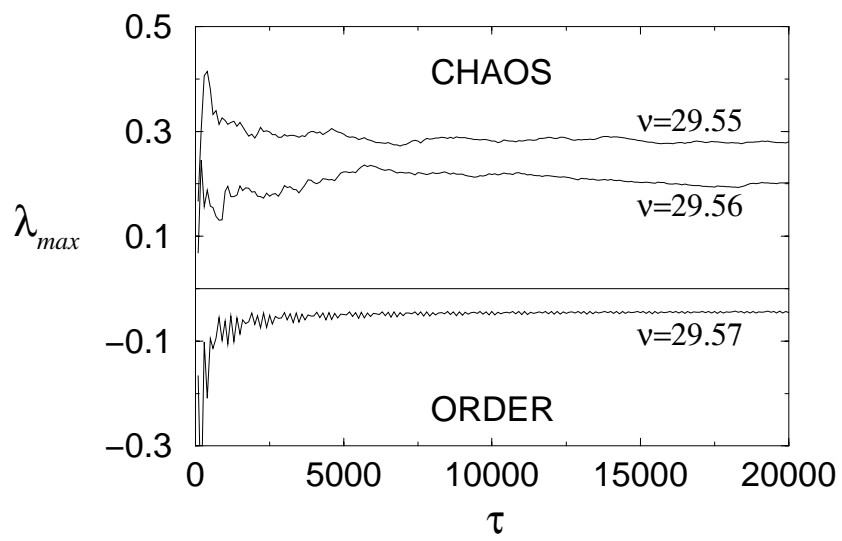

Fig. 5. The maximum Lyapunov exponent $\lambda_{\max }$ as a function of $\tau$ for the type-I Pomeau-Manneville intermittency route to chaos of Fig. 3.

saddle points. The head-on collision of the attractor with the period-2 saddle is clearly seen in Fig. 6 b.

Some examples of the time series for this crisis-induced intermittency are shown in Fig. 7. The corresponding power spectra and phase-space trajectories in a given time interval are displayed in Fig. 8. Figures $8 \mathrm{a}-\mathrm{c}$ show the variation of 

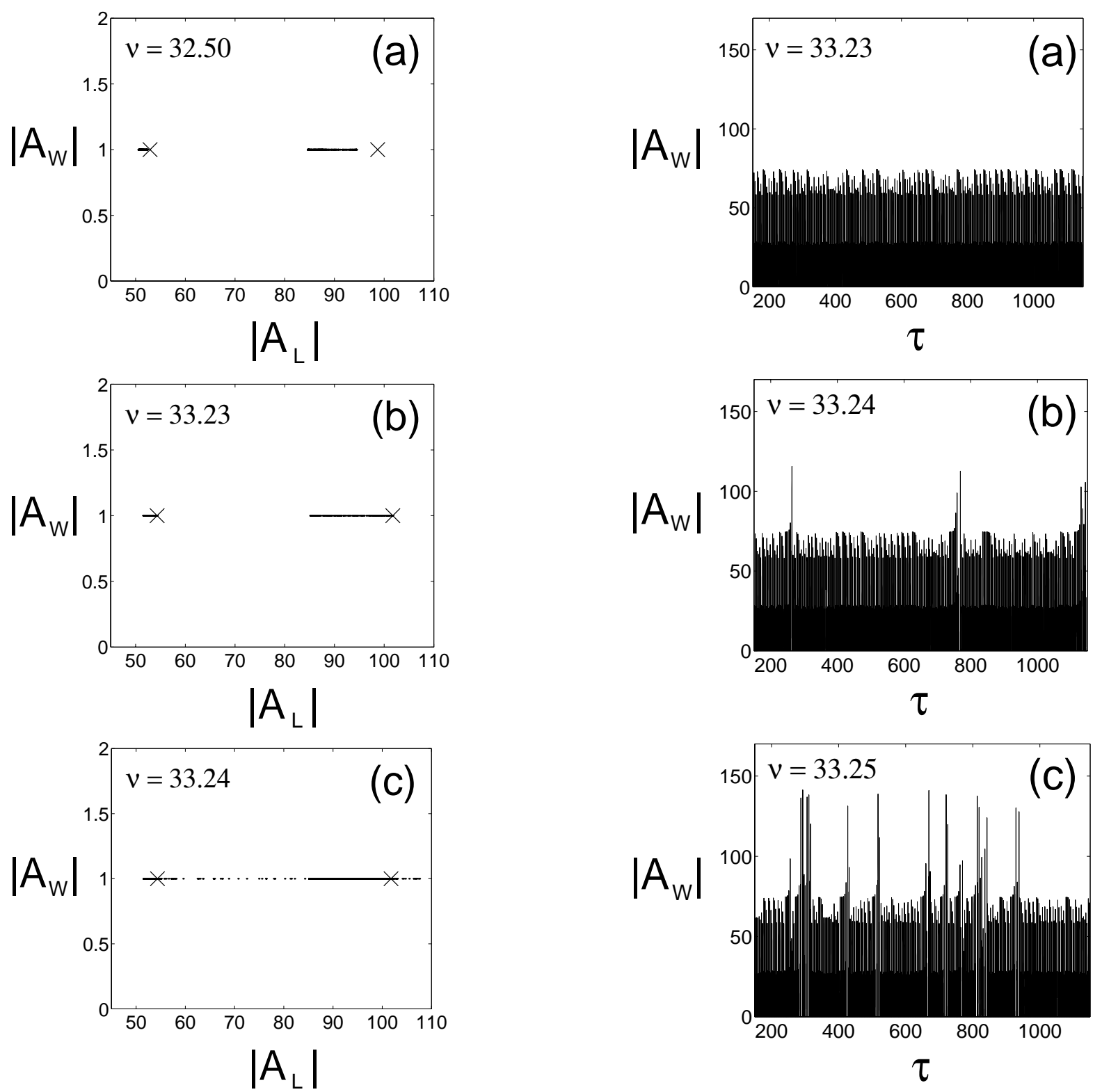

Fig. 6. Poincaré map $\left(\left|A_{L}\right|,\left|A_{W}\right|\right)$ for the interior crisis. (a) Before crisis at $v=32.50$, (b) at crisis at $v=v_{I C}=33.23$, (c) after crisis at $v=33.24$. The crosses denote the saddle points of the period-2 unstable periodic orbit.

the power spectra in log-log scale for $v=33.23$ (at crisis), $v=33.24$ (after crisis) and $v=33.25$ (further away from crisis). The slope of the power spectra at high frequencies decreases as $v$ increases. At crisis, the trajectory is confined to a small region of the phase space occupied by the weak chaotic attractor (Fig. 8d). After crisis, at $v=33.24$, the trajectory spends a long time in the region previously occupied by the weak chaotic attractor, after which it "bursts" to a wider region of phase space, before quickly going back to the neighborhood of the weak chaotic attractor (Fig. 8e). For $v=33.25$, strong chaotic bursts become more frequent in the time series (Fig. 7c); the trajectory now spends much less

Fig. 7. Time series of $\left|A_{W}\right|$ as a function of $\tau$ for the crisis-induced intermittency for (a) $v=33.23$, (b) $v=33.24$ and (c) $v=33.25$.

time in the vicinity of the weak chaotic attractor (Fig. 8f).

The maximum Lyapunov exponents for the time series of Fig. 7 are shown in Fig. 9. The increase in the system chaoticity as the control parameter $v$ is moved away from the crisis point is revealed by the maximum Lyapunov exponents.

\section{Discussions and conclusions}

Observational evidence of nonlinear coupling between whistler, Langmuir and Alfvén waves has been reported by several satellite and rocket experiments. A close correlation of auroral whistler waves and auroral Langmuir waves was 

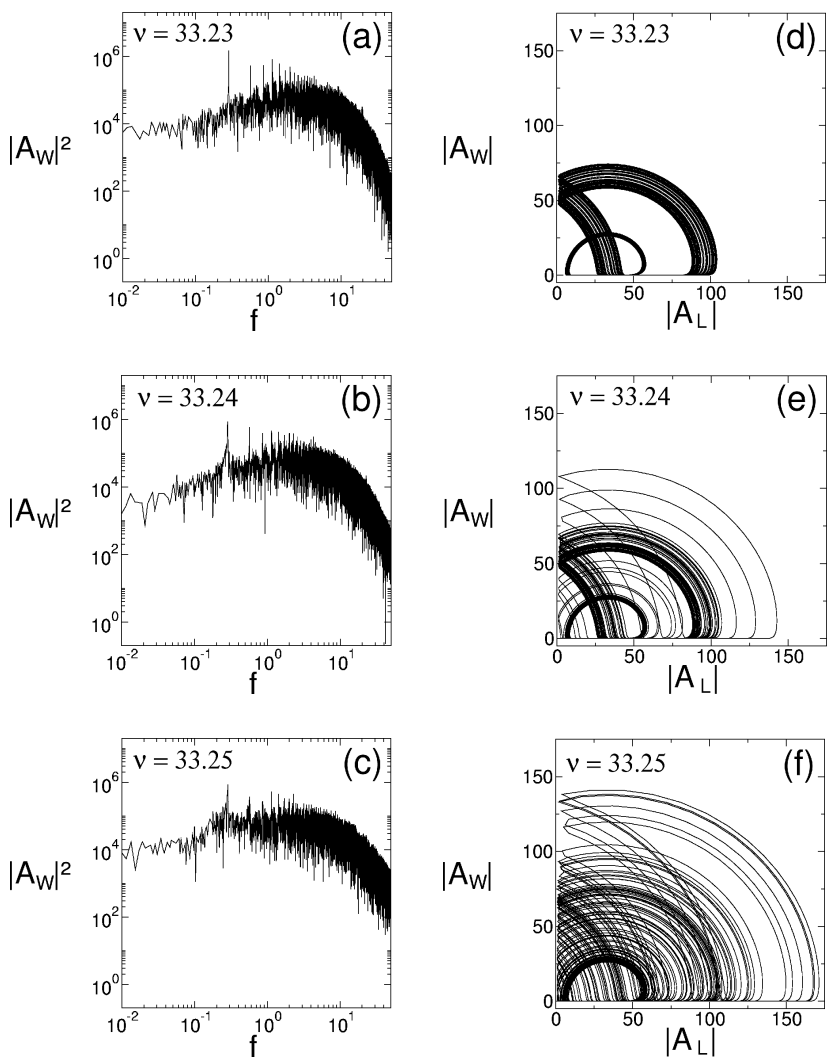

Fig. 8. Power spectra for (a) $v=33.23$, (b) $v=33.24$, (c) $v=33.25$; with the corresponding trajectories in the phase space $\left(\left|A_{L}\right|,\left|A_{W}\right|\right)$ in (d) to (f).

reported by Gurnett et al. (1983) and Beghin et al. (1989). A close correlation of auroral whistler waves and auroral Alfvén waves was reported by Gustafsson et al. (1990). In particular, simultaneous detection of whistler, Alfvén and Lagnmuir waves propagating along the auroral magnetic field lines was reported by Boehm et al. (1990), in connection with an intense flux of field-aligned electrons. The aforementioned observations render support for nonlinear interactions of whistler, Langmuir and Alfvén waves in space environment. In practice, for this three-wave interactions to occur, the waves must satisfy the phase-matching conditions. It was shown by Chian et al. (1994) that the phase-matching condition required for the resonant interactions of these three waves can be satisfied by Langmuir waves generated by a parametric decay of either a beam-driven Langmuir wave or a whistler wave.

In this paper, we have shown that chaos can appear in the nonlinear three-wave model proposed by Chian et al. (1994) for the auroral Langmuir-Alfvén-whistler (LAW) events. In particular, our nonlinear dynamical theory predicts that two types of intermittency can be observed: type-I PomeauManneville intermittency, due to a saddle-node bifurcation and crisis-induced intermittency, due to a global bifurcation involving the collision of a strange attractor with an unstable period orbit. Our results suggest that chaos is an intrinsic

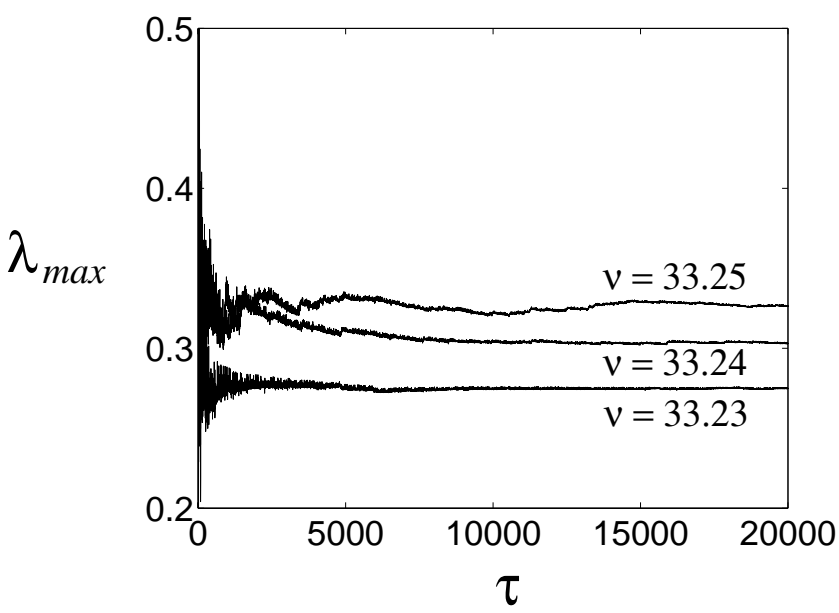

Fig. 9. The maximum Lyapunov exponent $\lambda_{\max }$ as a function of $\tau$ for the crisis-induced intermittency of Fig. 7.

behavior of magnetospheric radio emissions. The temporal time series of radio emissions from planets, the Sun and pulsars often exhibit quasi-periodic, intermittent or chaotic temporal patterns (Zhuravlev and Popov, 90; Isliker and Benz, 1994; Chian et al., 2000). Our theory shows that as the external parameters, such as the growth/damping parameters, are varied, the nonlinear dynamical behavior of radio emissions can change from periodic to chaotic temporal patterns via two different intermittency processes. According to our theory, the observed dynamical variations of radio emissions can be attributed to the dynamical changes in the physical parameters in the source region or along the propagation path of radio waves. A systematic analysis of the time series of magnetospheric radio waves based on the nonlinear dynamical techniques developed in this paper, such as the calculation of the maximum Lyapunov exponent of the observed time series, will enable the observers to identify the intermittent and chaotic features of magnetospheric radio emissions.

Acknowledgements. The authors wish to thank CNPq, FAPESP and AFOSR for support, and T. Thomas and T. Williams of Adelaide University for hospitality.

\section{References}

Alligood, K. T., Sauer, T. D., and Yorke, J. A.: Chaos: an Introduction to Dynamical Systems, Springer, New York, 1996.

Beghin, C., Rauch, J. L., and Bosqued, J. M.: Electrostatic plasma waves and HF auroral hiss generated at low altitude, J. Geophys. Res., 94, 1359-1378, 1989.

Boehm, M. H., Carlson, C. W., McFadden, J. P., Clemmons, J. H., and Mozer, F. S.: High-resolution sounding rocket observations of large-amplitude Alfvén waves, J. Geophys. Res., 95, $12157-$ $12171,1990$.

Chian, A. C.-L.: Non-thermal planetary radio emissions: theories and observations, in H.T. Duc Guyenne (ed.), Fusion and Astrophysical Plasmas, European Space Agency Publications, Noordwijk, 101-104, 1993. 
Chian, A. C.-L., Lopes, S. R., and Alves, M. V.: Generation of auroral whistler-mode radiation via nonlinear coupling of Langmuir waves and Alfvén waves, Astron. Astrophys., 290, L13L16, 1994.

Chian, A. C.-L., Borotto, F. A., and Gonzalez, W. D.: Alfvén intermittent turbulence driven by temporal chaos, Astrophys. J., 505, 993-998, 1998.

Chian, A. C.-L., Borotto, F. A., Lopes, S. R., and Abalde, J. R.: Chaotic dynamics of nonthermal planetary radio emissions, Planet. Sp. Sci., 48, 9-21, 2000.

Grebogi, C., Ott, E., and Yorke, J. A.: Crises, sudden changes in chaotic attractors, and transient chaos, Physica D, 7, 181-200, 1983.

Gurnett, D. A., Shawhan, S. D., and Shaw, R. R.: Auroral hiss, $\mathrm{Z}$ mode radiation, and auroral kilometric radiation in the polar magnetosphere: DE 1 observations, J. Geophys. Res., 88, 329340, 1983.

Gustafsson, G., André, M., Matson, L., and Koskinen, H.: On waves below the local proton gyrofrequency in auroral acceleration regions, J. Geophys. Res., 95, 5889-5904, 1990.

Isliker, H. and Benz, A. O.: On deterministic chaos, stationarity, periodicity and intermittency in coronal bursts and flares, Space Science Rev., 68, 185-192, 1994.

Lopes, S. R. and Chian, A. C.-L.: A coherent nonlinear theory of auroral Langmuir-Alfvén-Whistler (LAW) events in the planetary magnetosphere, Astron. Astrophys., 305, 669-676, 1996a.

Lopes, S. R. and Chian, A. C.-L.: Controlling chaos in nonlinear three-wave coupling, Phys. Rev. E, 54, 170-174, 1996 b.

Lopez-Rebollal, O., Sanmartin, J. R., and del Rio, E.: Sudden transition to chaos in plasma wave interactions, Phys. Plasmas, 5, 2861-2867, 1998.

Manneville, P. and Pomeau, Y.: Intermittency and the Lorenz model, Phys. Lett. A, 75, 1-2, 1979.

Meunier, C., Bussac, M. N., and Laval, G.: Intermittency at the onset of stochasticity in nonlinear resonant coupling processes, Physica D, 4, 236-243, 1982.

Wersinger, J. M., Finn, J. M., and Ott, E.: Bifurcations and strange behavior in instability saturation by nonlinear mode coupling, Phys. Rev. Lett., 44, 453-456, 1980.

Zhuravlev, V. I. and Popov, M. V.: Deterministic chaos in the microstructure of radio pulses from PSP 0809+74, Sov. Astron., 34, 377-381, 1990. 